\title{
CUCUMIS (CUCURBITACEAE) MUST INCLUDE CUCUMELLA, DICOELOSPERMUM, MUKIA, MYRMECOSICYOS, AND OREOSYCE: A RECIRCUMSCRIPTION BASED ON NUCLEAR AND PLASTID DNA DATA
}

\author{
HANNO SCHAEFER \\ Systematic Botany, Department of Biology, University of Munich, \\ Menzingerstraße 67, D-80638 Munich, Germany; \\ e-mail: hschaefer@1rz.uni-muenchen.de
}

\section{SUMMARY}

\begin{abstract}
Recent molecular studies have revealed that the genus Cucumis in its current circumscription is paraphyletic. To become monophyletic, Cucumis must include five genera of the subtribe Cucumerinae, namely Cucumella, Dicoelospermum, Mukia, Myrmecosicyos, and Oreosyce, already regarded as closely related by earlier workers based on morphology. The 19 species in the five genera are here formally transferred to Cucumis, resulting in 14 new combinations, two changes in status, and three new names (Cucumis indicus, $C$. kirkbrideana, and $C$. oreosyce). A description of the genus and a key to its 52 species are given, and the subgenera and sections are recircumscribed to reflect monophyletic groups. Subgenus Humifructus, with a haploid chromosome number of 12, contains only $C$. humifructus and C. hirsutus, while subgenus Cucumis, with $\mathrm{n}=12$ or $\mathrm{n}=7$, contains the remainder of the species. The latter is further divided into the sections Aculeatosi, Cucumella, $\mathrm{Cu}$ cumis, Metuliferi, and Sagittati.
\end{abstract}

Key words: Cucurbitaceae, Cucumis, Cucumella, Dicoelospermum, Mukia, Myrmecosicyos, Oreosyce.

\section{INTRODUCTION}

The following recircumscription of the genus Cucumis L. is based on molecular studies in which the author took part (Kocyan et al., in press; Renner et al., provisionally accepted). Although numerous specimens were studied during herbarium visits (E, FT, K, KUN, L, M, P, W, WAG, WU), species concepts here follow Kirkbride (1993, 1994) and De Wilde \& Duyfjes (2007). Phylogenetic analyses of plastid and nuclear DNA sequences (Kocyan et al., in press; Renner et al., provisionally accepted) show that Cucumis in its current circumscription is paraphyletic and needs to include five genera of the subtribe Cucumerinae Pax to become monophyletic, namely Cucumella Chiov., Dicoelospermum C.B. Clarke, Mukia Arn., Myrmecosicyos C. Jeffrey, and Oreosyce Hook.f. Based on leaf, flower and pollen morphology, De Wilde \& Duyfjes (2007) already combined Dicoelospermum with Mukia. All five of these genera were treated as close relatives of Cucumis in earlier morphological classifications of the Cucurbitaceae: Jeffrey (1980) placed them together with Cucumis in his broadly circumscribed tribe Melothrieae Endl. (34 genera) and mentions them explicitly as the closest relatives of 
Cucumis. In revised classifications (Jeffrey, 1990, 2005), he recognized a more narrowly circumscribed subtribe, Cucumerinae, which nevertheless still included Cucumeropsis Naudin, Melancium Naudin, Melothria L., Muellerargia Cogn., Posadaea Cogn., and Zehneria Endl. All but one of these genera are placed far from Cucumis based on the molecular data of Kocyan et al. (in press). The Australasian-African genus Muellerargia is the sister group to a monophyletic Cucumis (as recircumscribed formally herein).

\section{CUCUMIS}

Cucumis L. (1753) 1011. - Type: Cucumis sativus L. (lecto (Britton \& Wilson, 1925)).

Melo Mill. (1754) (no pagination). - Type: Cucumis melo L. (lecto (Swart, 1979)).

Mukia Arn. in Wight (1840) 50. - Melothria L. sect. Mukia (Arn.) Cogn. (1881) 622. - Type: Mukia scabrella (L.) Arn. (= Mukia maderaspatana (L.) M. Roem.).

Oreosyce Hook.f. (1871) 548. - Type: Oreosyce africana Hook.f.

Dicoelospermum C.B. Clarke (1879) 630 ('Dicaelospermum', correction Von Post \& Kuntze (1903)). - Type: Dicoelospermum ritchiei C.B. Clarke.

Hymenosicyos Chiov. (1911) 62. - Type: Hymenosicyos membranifolius (Hook.f.) Chiov. (=Oreosyce africana Hook.f.).

Cucumella Chiov. (1929) 183. - Type: Cucumella robecchii Chiov. (= Cucumella kelleri (Cogn.) C. Jeffrey).

Myrmecosicyos C. Jeffrey (1962) 357. - Type: Myrmecosicyos messorius C. Jeffrey.

Plants small to medium-sized climbers or trailers; annual or with perennial rootstock (rarely tubers) and herbaceous or perennial, woody shoots, usually hispid or scabridhairy. Tendrils present or rarely absent, solitary or rarely in groups of 5-8, simple or rarely bifid. Leaves simple, unlobed or palmately lobed, petiolate, margin entire or serrate. Flowers small to medium-sized, monoecious or rarely dioecious. Petals 5, yellow, disc free from the receptacle-tube. Male flowers solitary or in up to 19-flowered groups, sessile or pedicellate, rarely subtended by a bracteole; receptacle-tube infundibular to campanulate, sepals 5, rarely 4, small, long-triangular, linear, subulate or filiform; petals elliptic or (ob)ovate, free or united at base. Stamens 3, two double 2-thecous, 1 single 1-thecous, inserted near the middle of the receptacle-tube, filaments shorter than the anthers, glabrous; thecae lateral, straight (sometimes apically hooked) or sigmoid (triplicate), connective narrow, glabrous or papillate, puberulous to hairy, disc obconic or depressed globose, basal, free from the tube. Female flowers solitary or in fascicles of up to 6, usually separate from male flowers, pedicellate; ovary hairy, globose to oblong; ovules several to many, horizontal; perianth as in male flowers; style terete, thick, glabrous; stigma entire, sublobate or 3-lobed; lobes carnose, papillose, often with 1-9 finger-like projections on the margin; staminodes often present, three, subulate; disc annular, surrounding base of style, free from tube. Fruit fleshy, thin or rather firm-walled, indehiscent or rarely expelling seeds explosively, solitary or clusters of up to 6, subsessile or short-pedicelled, (sub)globose or ellipsoid, cylindrical, (ob)ovoid, or spindle-shaped, smooth and glabrous or pubescent or with dense to scattered fleshy spines, pustules or tubercles, that end in a hyaline bristle, sometimes beaked or fusiform, yellow, orange, red or greenish to brownish when ripe, often with longitudinal pale stripes, rarely maturing underground. Seeds small to medium-sized, few to many, globose or lenticular compressed, light-coloured, ovate or elliptic in outline, often ornamented, glabrous or rarely puberulent, margin often distinct, usually 
unwinged. Chromosome numbers $n=7,12$ (also polyploids with $n=24$ or 36 (Den Nijs \& Visser, 1985) and apparently aneuploid cultivars of $C$. melo with $\mathrm{n}=20$ or 22 (Chandola et al., 1965).

CUCUMIS subgenus Humifructus H. Schaef., nom. nov.

CuCumis series HuMifRuCTOSI J.H. Kirkbr. (1993) 77. - Type: Cucumis humifructus Stent (Species 1,2)

1. Cucumis hirsutus Sond. in Harv. \& Sond. (1862) 497. - Type: C.L.P. Zeyher 581 (lecto MEL, n.v. (Kirkbride, 1993)), South Africa, Inter Wonderfontyn \& Moojerivier.

2. Cucumis humifructus Stent (1927) 356. - Type: S.M. Stent s.n. (holo PRE, n.v.), South Africa, Transvaal, Pretoria, grown from seed sent by E.E. Galpin, Naboomspruit, Waterberg (Galpin M. 719), 13.02.1925.

CuCuMis subgenus CuCumis (Species 3-52)

CuCumis section CuCumis (Species 3-11)

3. Cucumis gracilis (Kurz) H. Schaef., comb. nov.

Basionym: Mukia maderaspatana (L.) M. Roem. var. gracilis Kurz (1877) 104. - Mukia scabrella (L.) Arn. var. gracilis (Kurz) C.B. Clarke (1879) 623. - Melothria maderaspatana (L.) Cogn. var. gracilis (Kurz) Cogn. (1881) 624. - Type: Wallich Cat. 6714 (iso K), Myanmar, Pagamew.

4. Cucumis hystrix Chakrav. (1952) 896. - Type: N.E. Parry 859 (holo K), India, Meghalaya, Garo Hills, Tura Mt, 04.11.1929; Paratype: Griffith 2554 (K), India, Arunachal Pradesh, Mishmee Hills.

5. Cucumis javanicus (Miq.) H. Schaef., comb. nov.

Basionym: Karivia javanica Miq. (1856) 661. - Melothria javanica Cogn. (1881) 625. - Mukia javanica (Miq.) C. Jeffrey (1969) 3, t. 3661. - Type: Horsfield s.n. (holo U, n.v.), Indonesia, Java.

6. Cucumis leiospermus (Wight \& Arn.) H. Schaef., comb. nov.

Basionym: Bryonia leiosperma Wight \& Arn. (1834) 345. - Mukia leiosperma (Wight \& Arn.) Wight (1840) 50. - Melothria leiosperma (Wight \& Arn.) Cogn. (1881) 622. - Type: Wight 1112 (lecto K (Jeffrey, 1969)), India, Madras, Palni Hills.

7. Cucumis maderaspatanus L. (1753) 1012

Synonym: Mukia maderaspatana (L.) M. Roem. (1846) 47. - Melothria maderaspatana Cogn. (1881) 623. - Type: t. 170, f. 2 in L., Plukenet, Almag. 1696, South Afrika (lecto (Meeuse, 1962); epitype: Herb. Plukenet 201 (BM, n.v.).

8. Cucumis melo L. (1753) 1011. - Type: Sweden. Plant cultivated at Uppsala (lecto LINN, sheet number 1152.8, n.v. (Meeuse, 1962)). 
9. Cucumis ritchiei (C.B. Clarke) H. Schaef., comb. nov.

Basionym: Dicoelospermum ritchiei C.B. Clarke (1879) 630 ('Dicaelospermum', correction Von Post \& Kuntze (1903)). - Mukia ritchiei (C.B. Clarke) W.J. de Wilde \& Duyfjes (2007). - Type: Ritchie 316 (lecto K (De Wilde \& Duyfjes, 2007)), India, Western.

Note - See Cucumis indicus (47) for a comment on nomenclature.

10. Cucumis rumphianus (Scheff.) H. Schaef., comb. nov.

Basionym: Melothria rumphiana Scheff. (1876) 25. - Mukia rumphiana (Scheff.) W.J. de Wilde \& Duyfjes (2007). - Type: Teijsmann 7496 (lecto n.v. (De Wilde \& Duyfjes, 2007)), Indonesia, Ternate.

11. Cucumis sativus L. (1753) 1012. - Type: J. Burser vol. 17, no. 97 (lecto UPS, n.v. (Ten Pas et al., 1985)), Europe. Cultivated plants.

CuCumis section ACuleatosi J.H. Kirkbr. (1993) 27. - Type: Cucumis dipsaceus Ehrenb. ex Spach (Species 12-35)

12. Cucumis aculeatus Cogn. (1896a) 209. - Type: G.L.A. Volkens 1972 (lecto BR, n.v. (Kirkbride, 1993)), Tanzania, Moshi Distr., Kilimanjaro, 1200 m, 08.03.1894.

13. Cucumis africanus L.f. (1782) 423. - Type: Hermann, Parad. bat. Tab. 134, 1698 (holo n.v.).

14. Cucumis anguria L. (1753) 1011. - Type: Sweden, plant cultivated at Uppsala (lecto LINN, sheet number 1152.6, n.v. (Kirkbride, 1993)).

15. Cucumis baladensis Thulin (1991) 541. - Type: Thulin, Hedrén \& Abdi Dahir 7464 (holo UPS, n.v.), Somalia, Shabeellaha Dhexe reg., 16 km N of Muqdisho along road to Balcad, 15.05.1990.

16. Cucumis canoxyi Thulin \& A.N. Al-Gifri (1994) 315. - Type: M. Thulin, Eriksson, Gifri, Langstroem 8299 (holo UPS, n.v.; iso Aden Univ., E, K), Yemen, Hadramaut.

17. Cucumis carolinus J.H. Kirkbr. (1993) 43. - Type: M.G. Gilbert \& M. Thulin 1116 (holo K), Kenya, Wajir Distr., 4 km SW Habaswein, 240 m, 27.04.1978.

18. Cucumis dipsaceus Ehrenb. ex Spach (1838) 211. - Type: G. Ehrenberg \& Hemprich s.n. (holo B†, lecto MPU, n.v. (Kirkbride, 1993)), Saudi Arabia, Wadi Kamme east of al-Qunfidha, Febr. 1825.

19. Cucumis ficifolius A. Rich. (1847) 294. - Type: R. Quartin-Dillon \& A. Petit s.n. (lecto P (Jeffrey, 1967)), Ethiopia, Sholoda.

20. Cucumis hastatus Thulin (1991) 535. - Type: M. Thulin, Hedrén \& Abdi Dahir 7765 (holo UPS, n.v.), Somalia, Bay reg., 52 km from Yaaq Bari Weyne to Buurhakaba, 23.05.1990. 
21. Cucumis heptadactylus Naudin (1859) 24. - Type: C. Zeyher 591 (lecto P (Kirkbride, 1993)), South Africa, Colesberg.

22. Cucumis insignis C. Jeffrey (1985) 209. - Type: Gilbert \& Jefford 4656 (holo $\mathrm{K})$, Ethiopia, Sidamo, $39 \mathrm{~km}$ from Yavello on new road to Agree Mariam, c. $1 \mathrm{~km}$ $\mathrm{S}$ of village of Sorupa, $1580 \mathrm{~m}$.

23. Cucumis jeffreyanus Thulin (1991) 539. - Type: W. Burger 3214 (holo K), Ethiopia, Harerge reg. N of Erer-Gota near Idorra, 31.08.1963.

24. Cucumis kalahariensis A. Meeuse (1962) 70. - Type: R. Story 5320 (lecto PRE, n.v. (Kirkbride, 1993)), South Africa, cultivated at Roodeplaat from seed collected in Namibia at Nama Pan (26.08.1955), Apr. 1957.

25. Cucumis meeusei C. Jeffrey (1965) 218. - Type: B. de Winter 3713 (holo K), Namibia, bed of Omuramba Omatako at Karahuwisa, 27.11.1955.

26. Cucumis messorius (C. Jeffrey) H. Schaef., comb. nov.

Basionym: Myrmecosicyos messorius C. Jeffrey (1962) 357. - Type: Bogdan 980 (holo K), Kenya, Nakuru Distr., Elmenteita, Soysambu Estate.

27. Cucumis myriocarpus Naudin (1859) 22. - Type: J.H. Bowker s.n. (lecto K (Kirkbride, 1993)), South Africa, Cape Province, Somerset.

28. Cucumis prolatior J.H. Kirkbr. (1993) 53. - Type: J.B. Gillett \& F.N. Gachathi 20478 (holo K), Kenya, Eastern, Kitui Distr., Nairobi-Garissa road, 3 km SW of Mwingi, 08.05.1974.

29. Cucumis prophetarum L. (1755) 33. - Type: F. Hasselquist s.n . (holo LINN, n.v.), Middle East.

30. Cucumis pubituberculatus Thulin (1991) 538. - Type: M. Thulin, Hedrén \& Abdi Dahir 7288 (holo UPS, n.v.), Somalia, Galgudud reg., Ceeldheer, just E of town, 07.05.1990.

31. Cucumis pustulatus Naudin ex Hook.f. (1871) 544. - Type: G.H. Schimper 835 (lecto K (Jeffrey, 1967)), Ethiopia, 31.06.1862.

32. Cucumis quintanilhae R. Fern. \& A. Fern. (1963) 269. - Type: R.B. Drummond 6025 (holo SRGH, n.v.), Zimbabwe, turn off to Limpopo Ranches, 10 miles north of Beitbridge, 25.03.1959.

33. Cucumis rigidus E. Mey. ex Sond. in Harv. \& Sond. (1862) 497. - Type: J.F. Drège s.n. (lecto K (Kirkbride, 1993)), South Africa, Cape Prov., on the Gariep River.

34. Cucumis thulinianus J.H. Kirkbr. (1993) 56. - Type: C.F. Hemming 2011 (holo K), Somalia, Northern reg., Erigavo, 1722 m, 05.10.1960. 
35. Cucumis zeyheri Sond. in Harv. \& Sond. (1862) 496. - Type: C. Zeyher 582 (lecto BM, n.v. (Kirkbride, 1993)) South Africa, Cape Prov., Gamka River, May 1840 .

CuCumis section SagitTati H. Schaef., sect. nov. - Type: Cucumis sagittatus Peyr. (Species: 36, 37)

36. Cucumis globosus C. Jeffrey (1965) 215. - Type: A. Harwood 19 (holo K), Tanzania, Mbeya Distr., about $1 \mathrm{~km}$ southeast of Ilomba local court, 27.02.1963.

37. Cucumis sagittatus Peyr. in Wawra \& Peyr. (1860) 567. - Type: H. Wawra 263 (lecto W, n.v. (Kirkbride, 1993)), Angola, prope Benguelam.

CuCumis section Metuliferi (J.H. Kirkbr.) H. Schaef., stat. nov. - Species $38-40$

Basionym: Cucumis ser. Metuliferi J.H.Kirkbr. (1993) 70. - Type: Cucumis metuliferus E. Mey. ex Naudin.

38. Cucumis metuliferus E. Mey. ex Naudin (1859) 10. - Type: C. Naudin s.n. (lecto P (Kirkbride, 1993)), France, Muséum Nationale d'Histoire Naturelle, 1857.

39. Cucumis rostratus J.H. Kirkbr. (1993) 72. - Type: J.P.M. Brenan 8875 (holo K), Nigeria, Benin Distr., Benin Div., Okomu Forest Reserve, compartment 69, 22.01.1948.

40. Cucumis sacleuxii Pailleux \& Bois (1890) 371. - Type: H.G. Faulkner 2865 (neo BR, n.v. (Kirkbride, 1993)), Tanzania, Zanzibar, Massazine, 15.07.1961.

CuCumis section CuCumella (Chiov.) H. Schaef., stat. nov. - Species 41-52

Basionym: Cucumella Chiov. (1929) 183. - Type: Cucumella robecchii Chiov.

41. Cucumis aëtheocarpus (C. Jeffrey) H. Schaef., comb. nov.

Basionym: Cucumella aëtheocarpa C. Jeffrey (1965) 215. - Type: M. Richards 17751 (holo K), Tanzania, Tunduru Distr., Tunduru-Masasi Road, 05.03.1963.

42. Cucumis asper Cogn. (1901) 882.

Synonym: Cucumella aspera (Cogn.) C. Jeffrey (1962) 349. - Type: Dinter 1447 (holo BR, n.v.), Namibia, Ababis, Hereroland, 16.02.1900.

43. Cucumis bryoniifolius (Merxm.) H. Schaef., comb. nov.

Basionym: Hymenosicyos bryoniifolia Merxm. (1953) 205. - Cucumella bryoniifolia (Merxm.) C. Jeffrey (1962) 350. - Type: R. Dehn 25'52" (holo M), Zimbabwe, Rusape, Aug. 1952.

44. Cucumis cinereus (Cogn.) H. Schaef., comb. nov.

Basionym: Kedrostis cinerea Cogn.(1901) 883. - Cucumella cinerea (Cogn.) C. Jeffrey (1962) 350. - Type: Dinter 1440 (holo Z, n.v.), Namibia, Giftkopje, Hereroland, 12.02.1900. 
45. Cucumis clavipetiolatus (J.H. Kirkbr.) H. Schaef., comb. nov.

Basionym: Cucumella clavipetiolata J.H. Kirkbr. (1994) 178. - Type: B. Nordenstam 3657 (holo S, n.v.), Namibia, Distr. of Omaruru, Brandberg, Orabeswand, 2000 m, 03.04.1964.

46. Cucumis engleri (Gilg) H. Schaef., comb. nov.

Basionym: Kedrostis engleri Gilg (1904) 359. - Cucumella engleri (Gilg) C. Jeffrey (1962) 350. - Type: Engler 1992 (lecto BR, n.v. (Kirkbride, 1994)), Kenya, Lake Nakuru, 30.08 .1902 .

47. Cucumis indicus H. Schaef., nom. nov.

Replaced synonym: Melothria ritchiei Chakrav. (1952) 898. - Cucumella ritchiei (Chakrav.) C. Jeffrey (1965) 215. - Type: Ritchie 67 (holo E, n.v.), India, Bombay Presidency, Savantvadi State, Ram Ghat.

Note - As both Dicoelospermum ritchiei C.B. Clarke and Cucumella ritchiei (Chakrav.) C. Jeffrey must be transferred to Cucumis, only one of them can keep its epithet. We decided to choose a new epithet for Cucumella ritchiei which was described in 1952, while Dicoelospermum ritchiei should keep the epithet because it was already described in 1879 .

48. Cucumis kelleri (Cogn.) H. Schaef., comb. nov.

Basionym: Oreosyce kelleri Cogn. (1896b) 822. - Cucumella kelleri (Cogn.) C. Jeffrey (1962) 350. - Type: Keller 111 (holo Z, n.v.), Ethiopia, Harrar, Webi Abdallah, 1891.

49. Cucumis kirkbrideana H. Schaef., nom. nov.

Replaced synonym: Cucumella jeffreyana J.H. Kirkbr. (1994) 168. - Type: L.C.C. Liebenberg 954 (holo K), Uganda, on roadside in grass country, Oct. 1929.

Note - A new epithet is required as jeffreyanus is preempted by $C$. jeffreyanus Thulin. The replacement name honours the botanist Joseph H. Kirkbride, who did groundbreaking biosystematic work on Cucumis.

50. Cucumis oreosyce H. Schaef., nom. nov.

Replaced synonym: Oreosyce africana Hook.f. (1871) 548. - Type: Mann 1285 (lecto K (here designated)), Equatorial Guinea, Bioko (Fernando Po).

Note - A new name is required as the epithet africanus is occupied by Cucumis africanus L.f.

51. Cucumis reticulatus (R. Fern. \& A. Fern.) H. Schaef., comb. nov.

Basionym: Cucumella reticulata R. Fern. \& A.Fern. (1969) 307. - Type: Milne-Redhead 4012 (holo K), Angola, Moxico, between Mumbala River and Namavumba River, 09.01.1938.

52. Cucumis silentvalleyi (Manilal, T. Sabu \& P. Mathew) H. Schaef., comb. nov.

Basionym: Cucumella silentvalleyi Manilal, T. Sabu \& P. Mathew (1985) 283 (as 'silentvalleyii'). - Type: Sabu SV 10662 (holo CAL, n.v.), India, Kerala, Palghat District, Silent Valley, Poochapara, 1370 m, 20.10.1982. 
KEY TO THE SPECIES OF CUCUMIS

(modified after Kirkbride, 1993, 1994)

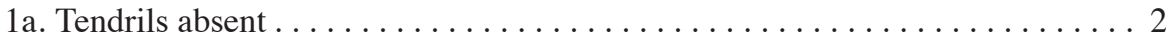

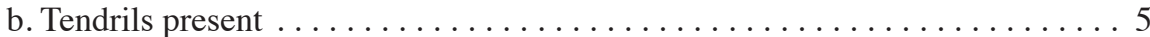

2a. Leaves deeply palmatisect, the lobes $1-2 \mathrm{~mm}$ wide . . . . . 26. C. messorius

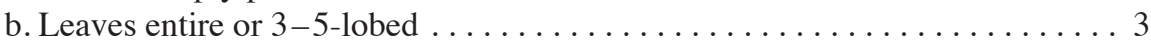

3a. Subshrub with erect stems $\ldots \ldots \ldots \ldots \ldots \ldots \ldots \ldots \ldots \ldots \ldots \ldots \ldots \ldots \ldots$. rigidus

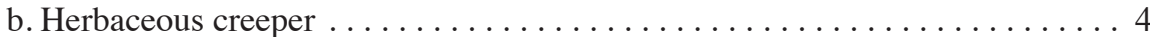

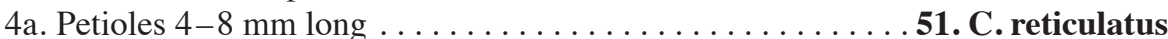

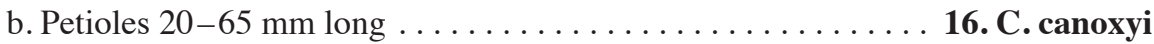

5a. Tendrils 5-8 per node. Fruit geocarpic, maturing below ground

2. C. humifructus

b. Tendrils solitary. Fruit maturing above ground $\ldots \ldots \ldots \ldots \ldots \ldots \ldots$

6a. At least some tendrils bifid $\ldots \ldots \ldots \ldots \ldots \ldots \ldots \ldots \ldots \ldots \ldots \ldots \ldots$ 22. . insignis

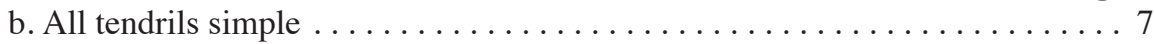

7a. Anther thecae straight or bent once at the apex into a short hook ...... 8

b. Anther thecae sigmoid . . . . . . . . . . . . . . . . . . . . 24

8a. Ovary and fruit bristly-tuberculate. Ripe fruit greenish, expelling seeds explo-

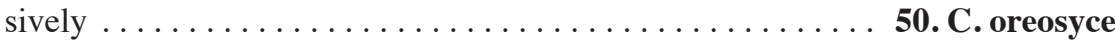

b. Ovary and fruit smooth, ribbed, aculeate-bristly or finely hairy. Fruit indehiscent

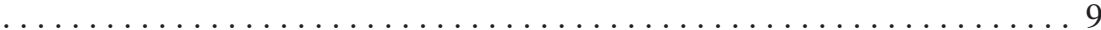

9a. Seeds globose, with faint central ridge, c. 3 per fruit. . . . . . . . 9. C. ritchiei

b. Seeds distinctly flattened, $>3$ per fruit $\ldots \ldots \ldots \ldots \ldots \ldots \ldots \ldots \ldots \ldots \ldots$

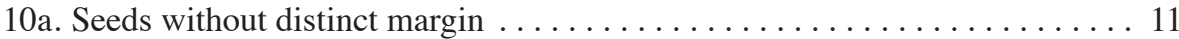

b. Seed margin distinct . . . . . . . . . . . . . . . . . . . . . . . . . . 19

11a. Leaves obtuse at base, without a basal sinus . . . . . . . . . 48. C. kelleri

b. Leaves cordate at base, with a basal sinus . . . . . . . . . . . . . 12

12a. Petioles with two different types of hairs $\ldots \ldots \ldots \ldots \ldots \ldots \ldots \ldots \ldots \ldots$

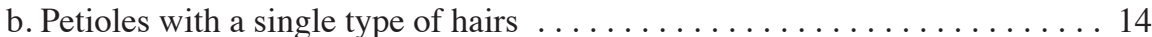

13a. Leaves entire, apex obtuse. Tendrils $10-25 \mathrm{~mm}$ long . . . . . . 46. C. engleri

b. Leaves pentalobate, apex acute. Tendrils $35-55 \mathrm{~mm}$ long 49. C. kirkbrideana

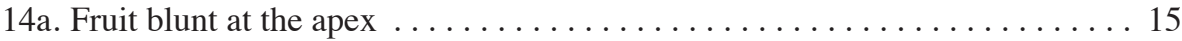

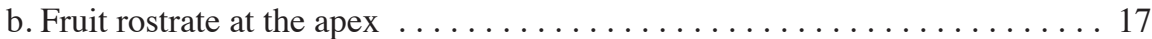

15a. Petioles claviform. Leaf-blade margin entire . . . . 45. C. clavipetiolatus

b. Petioles cylindrical. Leaf-blade margin serrate . . . . . . . . . . 16

16a. Plants herbaceous, hispidulous. Female flowers with six finger-like projections on the margin of the stigma . . . . . . bryoniifolius

b. Plants subshrubs, scabrous. Female flowers with three finger-like projections on the margin of the stigma $\ldots \ldots \ldots \ldots \ldots \ldots \ldots \ldots \ldots \ldots$ 42. C.asper

17a. Male flowers solitary . . . . . . . . aëtheocarpus

b. Male flowers in 3-19-flowered fascicles or racemes . . . . . . . . . . 18

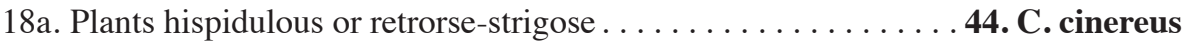

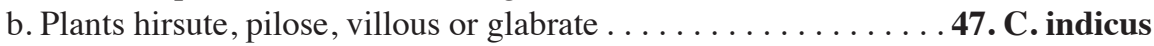

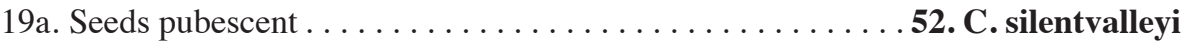

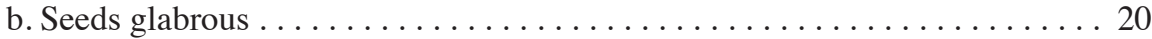


20a. Leaves ovate-oblong, usually longer than broad . . . . . . . 3. C. gracilis

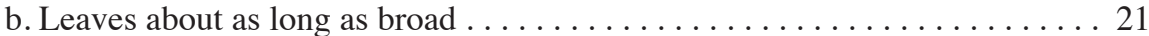

21a. Fruit ellipsoid, $20-35 \mathrm{~mm}$ long $\ldots \ldots \ldots \ldots \ldots \ldots \ldots$ 10. C. rumphianus

b. Fruit globose or ellipsoid, $<15 \mathrm{~mm}$ long $\ldots \ldots \ldots \ldots \ldots \ldots \ldots 22$

22a. Fruit ellipsoid, pericarp translucent. Seed faces flat . . . . . 5. C. javanicus

b. Fruit globose, pericarp not translucent. Seed faces convex . . . . . . . . . . 23

23a. Hairs of petiole spreading or recurved. Seed faces smooth or low-warted, the margin separated by a groove $\ldots \ldots \ldots \ldots \ldots \ldots \ldots$ 6. C. leiospermus

b. Hairs of petiole spreading or upcurved. If seed faces smooth, then without a distinct margin $\ldots \ldots \ldots \ldots \ldots \ldots \ldots \ldots \ldots \ldots \ldots \ldots \ldots \ldots \ldots \ldots \ldots \ldots$. C. maderaspatanus

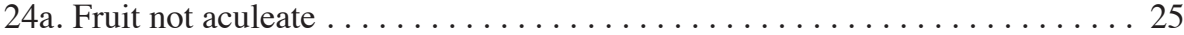

b. Fruit aculeate . . . . . . . . . . . . . . . . . . . . . . 30

25a. Seeds $<2.5 \mathrm{~mm}$ wide $\ldots \ldots \ldots \ldots \ldots \ldots \ldots \ldots \ldots \ldots \ldots \ldots \ldots \ldots$ 37. . sagittatus

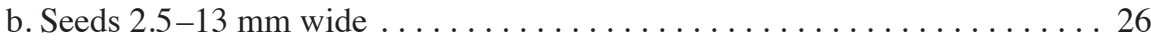

26a. Fruit rostrate at apex $\ldots \ldots \ldots \ldots \ldots \ldots \ldots \ldots \ldots \ldots \ldots \ldots \ldots$. rostratus

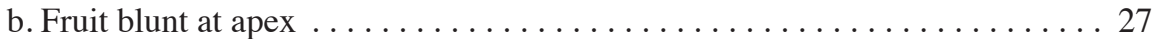

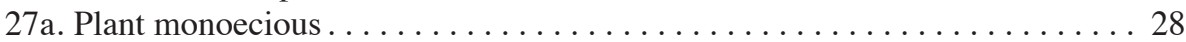

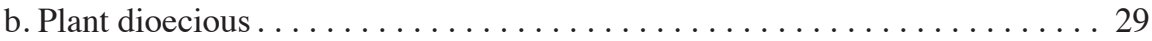

28a. Leaf-blade lobe elliptic, oblong or ovate in outline. Corolla tube $0.8-2.8 \mathrm{~mm}$ long ............ melo

b. Leaf-blade lobe broadly triangular in outline. Corolla tube $3.4-6.5 \mathrm{~mm}$ long . .

11. C. sativus

29a. Internodes 25-40 mm long. Leaves hirsute. Male flowers in groups of up to 11, pedunculate $\ldots \ldots \ldots \ldots \ldots \ldots \ldots \ldots \ldots \ldots \ldots \ldots \ldots \ldots \ldots \ldots \ldots \ldots$. hirsutus

b. Internodes 50-70 mm long. Leaves pilose. Male flowers solitary, sessile . . . . .

15. C. baladensis

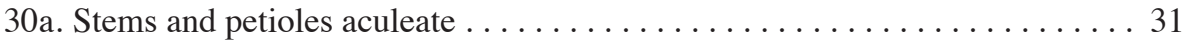

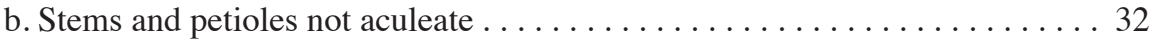

31a. Petioles glabrous. Fruit pedicel glabrate. Fruit $60-80 \mathrm{~mm}$ long 12. C. aculeatus

b. Petioles hispidulous. Fruit pedicel hispidulous. Fruit $25-40 \mathrm{~mm}$ long . . . . . . .

19. C. ficifolius

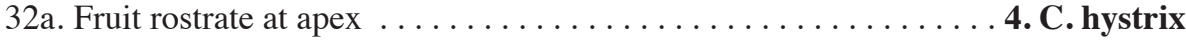

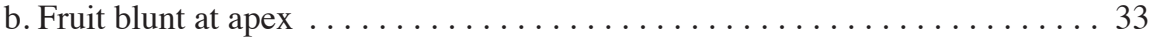

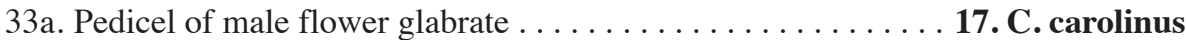

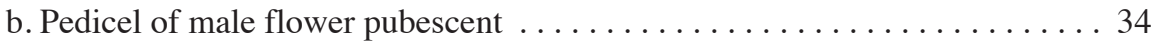

34a. Fruit aculei $20-22 \mathrm{~mm}$ long $\ldots \ldots \ldots \ldots \ldots \ldots \ldots \ldots \ldots \ldots \ldots \ldots \ldots \ldots$ 28. prolatior

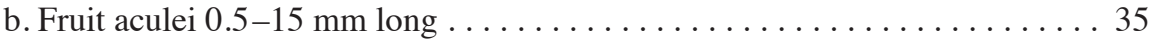

35a. Pedicel of female flower and fruit pedicel flaring upwards from a narrower base

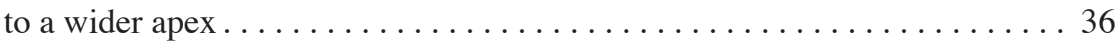

b. Pedicel of female flower and fruit pedicel cylindrical . . . . . . . . . 38

36a. Annual, woody rootstock absent $\ldots \ldots \ldots \ldots \ldots \ldots \ldots \ldots$ 14. C. anguria

b. Perennial with woody rootstock $\ldots \ldots \ldots \ldots \ldots \ldots \ldots \ldots \ldots \ldots \ldots$

37a. Leaves antrorse-strigose or scabrous. Pedicel of female flower $8-13 \mathrm{~mm}$ long. .

25. C. meeusei

b. Leaves hispidulous. Pedicel of female flower $15-45 \mathrm{~mm}$ long 
38a. Plant dioecious . . . . . . . . . . . . . . . . . . . . . 39

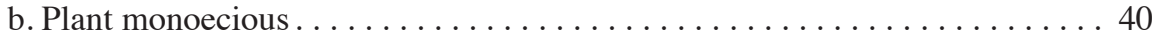

39a. Woody rootstock and tubers present. Nodes not geniculate. Leaf lobes 5-30 mm wide

24. C. kalahariensis

b. Woody rootstock and tubers absent. Nodes geniculate. Leaf lobes $1-5 \mathrm{~mm}$ wide

21. C. heptadactylus

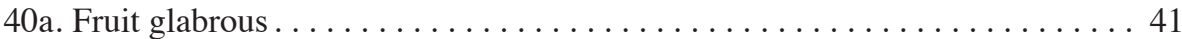

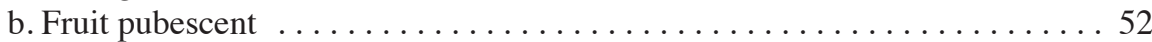

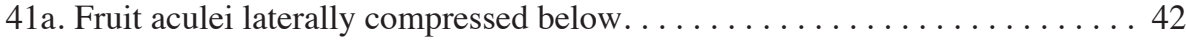

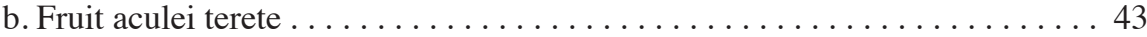

42a. Stems and petioles scabrous. Petioles $20-85 \mathrm{~mm}$ long. Male flowers in 5-10flowered racemes. Fruit ellipsoid or cylindrical . . . . . 13. C. africanus

b. Stems and petioles retrorse-strigose. Petioles 5-15 mm long. Male flowers solitary.

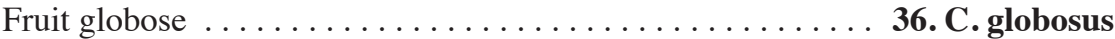

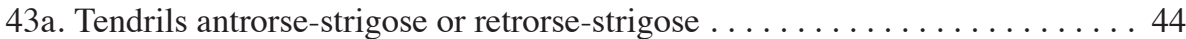

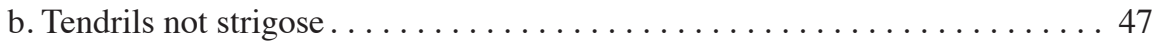

44a. Female corolla puberulent inside. Petiole with 3 different types of hairs in different

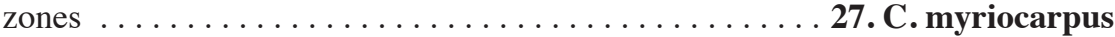

b. Female corolla glabrous inside. Petiole with 1 type of hairs . . . . . . . . 45

45a. Annual. Pedicel of female flower $55-65 \mathrm{~mm}$ long . . . . . 32. C. quintanilhae

b. Perennial. Pedicel of female flower $<40 \mathrm{~mm}$ long . . . . . . . . . . 46

46a. Male corolla glabrous inside, the lobes 2.4-5.6 mm long. Leaves hispidulous . .

29. C. prophetarum

b. Male corolla puberulent inside, the lobes c. $10 \mathrm{~mm}$ long. Leaves antrorse-

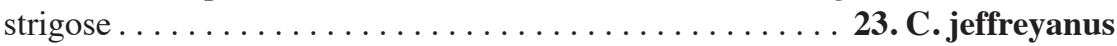

47a. Seeds c. $8.5 \mathrm{~mm}$ long, c. $5.5 \mathrm{~mm}$ wide and c. $3.7 \mathrm{~mm}$ thick . . 36. C. globosus

b. Seeds $<8 \mathrm{~mm}$ long, $2-4 \mathrm{~mm}$ wide, $0.9-1.6 \mathrm{~mm}$ thick . . . . . . . . . 48

48a. Woody rootstock absent. Female sepals 4.8-11 mm long. Female corolla glabrous

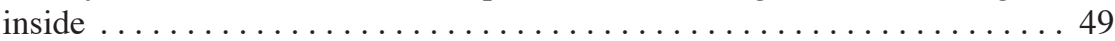

b. Woody rootstock present. Female sepals $1.2-4.2 \mathrm{~mm}$ long. Female corolla pubes-

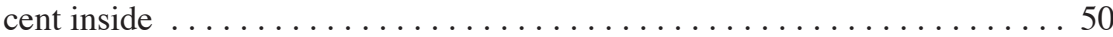

49a. Male and female corolla lobes obovate in outline, acute at apex. Female pedicel 5-15 mm long. Fruit aculei 4-6.4 mm long. Seeds $4-5 \mathrm{~mm}$ long, c. $2 \mathrm{~mm}$

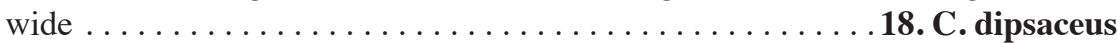

b. Male and female corolla lobes elliptic in outline, obtuse at apex. Female pedicel 55-65 mm long. Fruit aculei 8.5-13 mm long. Seeds 6-6.5 mm long, 2.5-3 mm

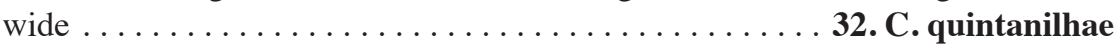

50a. Corolla lobes $8-12 \mathrm{~mm}$ long, scabrous outside. Seeds $<4 \mathrm{~mm}$ long $\ldots \ldots \ldots$.

30. C. pubituberculatus

b. Corolla lobes 2.4-5.6 mm long, hispidulous outside. Seeds $4.3-6 \mathrm{~mm}$ long 51

51a. Leaf base obtuse, truncate or cordate. Fruit green with paler longitudinal stripes

29. C. prophetarum

b. Leaf base sagittate. Fruit monocoloured, yellow . . . . . . 34. C. thulinianus

52a. Seeds ovate, 3.3-4 mm wide. Female corolla lobes $5.1-12 \mathrm{~mm}$ wide . . . . . .

38. C. metuliferus

b. Seeds elliptic, 2-3.1 mm wide. Female corolla lobes $2-4 \mathrm{~mm}$ wide . . . . 53 
53a. Stems and petioles antrorse-strigose or retrorse-strigose. Central leaf-blade lobe pinnatifid. Aculei on fruit $1.3-13 \mathrm{~mm} \ldots \ldots \ldots \ldots \ldots \ldots 4 \ldots \ldots \ldots$

b. Stems and petioles hispidulous, pilose, or scabrous. Central leaf-blade lobe entire.

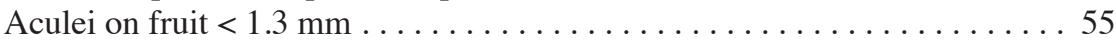

54a. Male and female corolla glabrous inside, male corolla lobes obtuse at apex ....

29. C. prophetarum

b. Male and female corolla puberulent inside, male corolla lobes acute at apex... .

35. C. zeyheri

55a. Annual. Stems, petioles, leaves, pedicels of female flowers and fruits with breakaway hairs. Male corolla lobes $4.8-6 \mathrm{~mm}$ wide . . . . . . . 40. C. sacleuxii

b. Perennial. Stems, petioles, leaf blades, and pedicels of female flowers and fruit with persistent hairs. Male corolla lobes $1.3-2 \mathrm{~mm}$ wide. . . . . . . . . 56

56a. Leaf-lobes elliptic in outline. Leaf-blades hispidulous . . 29. C. prophetarum

b. Leaf-lobes ovate in outline. Leaf-blades scabrous . . . . . . 20. C. hastatus

\section{ACKNOWLEDGEMENTS}

The author would like to thank Susanne Renner, Brigitta and Willem de Wilde, and Joseph H. Kirkbride for comments on earlier drafts of this manuscript. Work for this project was supported by the German National Science Foundation (RE 603/3-1).

\section{REFERENCES}

Britton, N.L. \& P. Wilson. 1925. Scientific survey of Porto Rico and the Virgin Islands 6. Bot. Porto Rico \& Virgin Islands: 159-316. Academy of Sciences, New York.

Chakravarty, H.L. 1952. New finds of Indian Cucurbitaceae. J. Bombay Nat. Hist. Soc. 50: 892 901.

Chandola, R.P., M.P. Bhatnagar \& I. Tokuta. 1965. Cytological studies of some varieties of musk melon with special reference to their relationship. Cytologia 30: 252-259.

Chiovenda, E. 1911. Plantae novae vel minus notae e regione aethiopica. Ann. Bot. (Rome) 9: $51-85$.

Chiovenda, E. 1929. Fl. Somala 1: 1-436. Pubblicazione a cura del ministero delle colonie, Roma, Modena, Siena.

Clarke, C.B. 1879. Cucurbitaceae. In: J.D. Hooker, The Flora of British India 2: 604-635. Reeve \& Co., London.

Cogniaux, A. 1881. Cucurbitaceae. In: A.L.L.P. \& A.C.P. de Candolle, Monogr. Phan. 3: 325-951. Masson, Paris.

Cogniaux, A. 1896a. Cucurbitaceae africanae. Bot. Jahrb. Syst. 21: 207-211.

Cogniaux, A. 1896b. Cucurbitaceae. In: H. Schinz, Beiträge zur Kenntnis der afrikanischen Flora V. Bull. Herb. Boissier 4: 809-846.

Cogniaux, A. 1901. Cucurbitaceae. In: H. Schinz, Beiträge zur Kenntnis der afrikanischen Flora. Bull. Herb. Boissier, sér. 2, 1: 853-889.

De Wilde, W.J.J.O. \& B.E.E. Duyfjes. 2007. Mukia Arn. (Cucurbitaceae) in Asia, in particular in Thailand. Thai Forest Bull., Bot. 34: 38-52.

Den Nijs, A.P.M. \& D.L. Visser. 1985. Relationships between African species of the genus Cucumis L. estimated by the production, vigour and fertility of F1 hybrids. Euphytica 34: 279-290.

Fernandes, R. \& A. Fernandes. 1963. Cucumis quintanilhae sp. nov. Revista Biol. (Lisbon) 3, 2-4: 269.

Fernandes, R. \& A. Fernandes. 1969. Cucurbitaceae africanae novae II. Bol. Soc. Brot., sér. 2, 43: 307-309.

Gilg, E. 1904. Cucurbitaceae africanae II. Bot. Jahrb. Syst. 34: 343-367. 
Harvey, W.H. \& O.W. Sonder. 1862. Flora Capensis 2. Cucurbitaceae: 482-498. Hodges, Smith \& Co., Dublin.

Hooker, J.D. 1871. Cucurbitaceae. In: D. Oliver (ed.), Flora of Tropical Africa 2: 521-569.

Jeffrey, C. 1962. Notes on Cucurbitaceae, including a proposed new classification of the family. Kew Bull. 15: 337-371.

Jeffrey, C. 1965. Further notes on Cucurbitaceae. Kew Bull. 19: 215-223.

Jeffrey, C. 1967. Cucurbitaceae. In: E. Milne-Redhead \& R.M. Polhill (eds.), Flora of Tropical East Africa. Whitefriars Press, London.

Jeffrey, C. 1969. The genus Mukia in Asia, Malesia and Australasia. Hooker's Icon. Pl. 5, 7, 3 : $1-12$.

Jeffrey, C. 1980. A review of the Cucurbitaceae. J. Linn. Soc., Bot. 81: 233-247.

Jeffrey, C. 1985. Further notes on Cucurbitaceae: VII Preliminary to the Flora of Ethiopia. Kew Bull. 40: 209-211.

Jeffrey, C. 1990. Systematics of the Cucurbitaceae: An overview. In: D.M. Bates, R.W. Robinson \& C. Jeffrey (eds.), Biology and utilization of the Cucurbitaceae 3-9, 449-463. Cornell University Press, Ithaca.

Jeffrey, C. 2005. A new system of Cucurbitaceae. Bot. Zhurn. (St. Petersburg) 90: 332-335.

Kirkbride Jr., J.H. 1993. Biosystematic monograph of the genus Cucumis (Cucurbitaceae). Parkway Publishers, Boone, NC.

Kirkbride Jr., J.H. 1994. Revision of Cucumella (Cucurbitaceae, Cucurbitoideae, Melothrieae, Cucumerinae). Brittonia 46: 161-186.

Kocyan, A., L.-B. Zhang, H. Schaefer \& S.S. Renner. In press. A multi-locus chloroplast phylogeny for the Cucurbitaceae and its implications for character evolution and classification. Molec. Phylogenet. Evol.

Kurz, S. 1877. Contributions towards a knowledge of the Burmese flora. J. Asiat. Soc. Bengal 46, 2: $95-106$.

Linnaeus, C. 1753. Species Plantarum. Impensis Laurentii Salvii, Stockholm.

Linnaeus, C. 1755. Centuria 1 Plantarum. Impensis Laurentii Salvii, Stockholm.

Linnaeus f., C. 1782 ('1781'). Supplementum Plantarum Systematis Vegetabilum. Braunschweig.

Manilal, K.S., T. Sabu \& P. Mathew. 1985. A new species of Cucumella Chiov. (Cucurbitaceae) from India. Acta Bot. Indica 13, 2: 283-284.

Meeuse, A.D.J. 1962. The Cucurbitaceae of southern Africa. Bothalia 8: 1-111.

Merxmüller, H. 1953. Neue Sippen aus Süd-Rhodesia. Mitt. Bot. Staatssamml. München 1, 6: 196-208.

Miller, P. 1754. The gardener's dictionary. Abr. ed. 4. London.

Miquel, F.A.W. 1856. Flora Indiae Batavae 1, 1: 652-683. Van der Post, Amsterdam.

Naudin, C.V. 1859. Essaie d'une monographie des espèces et des variétés du genre Cucumis. Ann. Sci. Nat., Bot. sér. 4, 11: 5-87.

Pailleux, A. \& D.G.J.M. Bois. 1890. Cultures expérimentales en 1889. Concombre de Mandéra. Cucumis sacleuxii, sp. nov. Bull. Soc. Natl. Acclim. France 37, 8: 371-373.

Renner, S.S., H. Schaefer \& A. Kocyan. Phylogenetics of Cucumis: Broad taxon sampling in Cucurbitaceae shows that C. sativus (cucumber) belongs in an Australian/Asian clade far from African melons. (Evolutionary Biology, accepted pending revision).

Richard, A. 1847. Tentamen florae abyssinicae 1. Paris.

Roemer, M.J. 1846. Peponiferarum. Syn. Monogr. 2: 1-118. Landes-Industrie-Comptoirs, Weimar.

Scheffer, R.H.C.C. 1876. Énumeration des plantes de la Nouvelle-Guinée, avec déscription des espèces nouvelles. Ann. Jard. Bot. Buitenzorg 1: 1-60.

Spach, E. 1838. Histoire naturelle des végétaux 6. Paris.

Stent, S.M. 1927. An undescribed geocarpic plant from South Africa. Bothalia 2, 1b: 356-359.

Swart, J.J. 1979. Melo. In: E.R. Farr, J.A. Leussink \& F.A. Stafleu (eds.), Index nominum genericorum (plantarum). Vol. 2. Bohn, Scheltema \& Holkema, Utrecht.

Ten Pas, H.N., J.W.P. Schoenaker, E.H. Oost \& C.E. Jarvis. 1985. Re-lectotypification of Cucumis sativus L. Taxon 34: 288-293. 
Thulin, M. 1991. New species of Cucumis (Cucurbitaceae) from northeast tropical Africa. Nordic J. Bot. 11: 535-542.

Thulin, M. \& A.N. Al-Gifri. 1994. Cucumis canoxyi (Cucurbitaceae) - a new species from Yemen. Nordic J. Bot. 14: 315-317.

Von Post, T. \& O. Kuntze. 1903. Lexicon Generum Phanerogamarum. Deutsche Verlags-Anstalt, Stuttgart.

Wawra, H. \& J.J. Peyritsch. 1860. Sertum benguelense. Aufzählung und Beschreibung der auf der Expeditionsfahrt S.M. Corvette 'Carolina' an der Küste von Benguela von dem Corvetten-Arzt Dr. Heinrich Wawra gesammelten Pflanzen. Sitzungsber. Kaiserl. Akad. Wiss. Math.-Naturwiss. Cl. 38: 543-585.

Wight, R. 1840. Remarks on the fruit of the natural order Cucurbitaceae. Madras J. Lit. Sci. 12: 48-54.

Wight, R. \& G.A.W. Arnott. 1834. Prodromus Florae Peninsulae Indiae Orientalis 1: 340-351. Parbury, Allen \& Co., London. 